\title{
Automating Documentation: A critical perspective into the role of artificial intelligence in clinical documentation
}

\author{
Matt Willis ${ }^{1[0000-0002-9120-7319]}$ and Mohammad Hossein Jarrahi2[0000-0002-5685-7156] \\ ${ }^{1}$ Oxford Internet Institute, University of Oxford. OX1 3JS, UK \\ ${ }^{2}$ University of North Carolina, Chapel Hill, NC 27599, USA
}

\begin{abstract}
The current conversation around automation and artificial intelligence technologies creates a future vision where humans may not possibly compete against intelligent machines, and that everything that can be automated through deep learning, machine learning, and other AI technologies will be automated. In this article, we focus on general practitioner documentation of the patients' clinical encounter, and explore how these work practices lend themselves to automation by AI. While these work practices may appear perfect to automate, we reveal potential negative consequences to automating these tasks, and illustrate how AI may render important aspect of this work invisible and remove critical thinking. We conclude by highlighting the specific features of clinical documentation work that could leverage the benefits of human-AI symbiosis.
\end{abstract}

Keywords: Automation, Artificial Intelligence, Healthcare, Clinical Documentation

\section{Introduction}

Artificial Intelligence (AI) is lauded for its promising potentials to transform work, from robots performing a variety of services to algorithms performing knowledge-intensive work practices in healthcare [1]. In this paper, we take a measured approach grounded in extensive ethnographic fieldwork in healthcare to examine the complex and contextual documentation practices of general doctors that requires the ability to overcome not technical challenges, but also social ones. This paper builds on an empirical investigation of the Primary Care service sector of the National Health Service (NHS) in England, and presents a detailed account of clinical documentation work, critical work that happens after a provider sees a patient. As Bansler et al [2] note: "clinical notes form the core of the medical record" (p.503). While some argue this work can be largely automated [3, 4], in this paper we ask the key question: just because something can technically be automated, should it necessarily be? 


\subsection{The need for automation in healthcare}

The NHS in England is under remarkable pressure to improve services, cut costs, and address low staff morale [5]. These challenges must be met under the backdrop of the longest financial crunch in the history of the NHS with a dramatic increase in service demand and use, lagging performance in mental health services, staff shortages, ageing population, and increased wait times [6].

The British Medical Association (BMA) also designed a survey that was conducted by ICM Research to survey General Practitioners (GPs) in England. Of the over 5,000 GP respondents, $84 \%$ describe their workload as excessive and prevents the safe delivery of care; only one in ten said their workload in manageable and allows for quality and safe care. This has caused $38 \%$ of partners to consider closing their lists to better manage workload [7].

A similar survey on GP worklife from the Policy Research Unit in Commissioning and the Healthcare System has measures on stressors and job satisfaction that are relevant to this discussion on workload and care. Of the 14 stressors the survey measures, the most stressful are workload and meeting requirements of external bodies. The least stressful are finding a locum and emergency call interruptions. However, every single stressor was at its highest point (on a 5-point scale) since the beginning of the National GP Worklife Survey in 1998. Job satisfaction declined from 4.5 points in 2012 to 4.1 points in 2015 , with the most significant areas for dissatisfaction being hours worked and remuneration [8].

This review of multiple reports suggests a crucial sector of the NHS that is burntout, over-worked, dissatisfied, and at a disadvantage for resources, staff, and time. It is easy to see how automation and other appropriate and strategic implementations of intelligent systems could play a role in reducing stress and increase effectiveness by providing more time to primary care staff and doctors.

In this paper, we focus on one specific area of work in primary care that can benefit from the application of AI to address many of the previously noted ailments: clinical documentation. Little research has been done on documentation work and how it may benefit from AI.

\subsection{Documentation and automation}

Automation of work using intelligent systems has long been an object of academic research and practical concerns [9]. Automation technologies are expensive investments and take time to develop and implement into an organization. It is not the best economical decision to automate a task that is performed once a month for an hour. Automation provides the greatest advantages when it is applied to a task that is frequent or time consuming, is important, and requires a degree of repetitiveness in the work task. Many may see clinical documentation as a fertile ground for automation because it fits three hallmarks of tasks that benefit most from automation in healthcare [10].

First, it is a task that every clinician must complete, and with a sense of urgency. It is routinely described as good medical practice to document the patient consultation as near the end of the appointment as possible. Documentation of all patient encounters at the end of the day is an unwanted situation and, in many cases, it is impossible with the 
number of patients seen over the duration of a clinic. Second, documentation takes attention and time [3]. Although the amount of necessary attention depends on the patient's unique condition and the relationship between the patient and the provider, even brief notes require the clinician to accurately describe brief encounters [11]. Third, clinicians are typically given no time to document. As important as proper documentation is to the practice of medicine, a clinician typically documents during the amount of time it takes a patient to leave the examination room and the next patient to walk into the examination room from the waiting room with no time buffer. During a full clinic, the tempo of documentation and patient examination can be challenging and exhausting.

Clinical documentation constitutes a large portion of doctors' administrative tasks; doctors may need to spend up to $55 \%$ of their workday to generate notes and to review patient's records [3]. Studies suggest workload caused by clinical documentation is an important barrier to effective patient care [12]. Using existing technologies, vendors of electronic health record systems and researchers have long looked for ways to facilitate the process by pulling in structured data/format into doctor's note to reduce duplicate work [13]. Recently some researchers have advocated for the application of AI systems to streamline and automate documentation tasks in healthcare [3,4], for example, by using natural language processing to dissect patient-doctor conversation and create notes [14]. However, incorporation of smart technologies into clinical documentation practices has been a controversial topic since research presents them as a web of complex work practices with institutional, social and situated dimensions [2].

Given the centrality of documentation work in healthcare and the new wave of computerization and AI that provide promising opportunities for automating work, we ask the research question: What problems do general practitioners clinical documentation work practices create for automation by AI?

\section{$2 \quad$ Method}

To understand detailed work in a complex and fast paced clinical environment we employ observational fieldwork to gather empirical data. The first author observed six primary care practices across England spending on average a week at each practice (located in the areas of: Oxfordshire, Yorkshire, Berkshire, Surrey, and West-Midlands). The patient registry of observed practices ranges from the smallest of 5,000 to the largest of 24,000, with an average of about 11,500 patients per surgery.

The focus of the fieldwork was to understand the situated work practices of each occupational type at each primary care practice for a larger research project. The observation of each occupation does not mean every staff member in the practice, but the occupations at that practice. For example, if there are 12 receptionists working at the practice the field researcher does not observe and interview all 12, but a sample of them, depending on availability and consent, till the work of a receptionist is clearly understood and can be accurately represented in the data. In total this resulted in 65 sessions of observation ranging from 45 minutes to an hour and 20 minutes in length. The observed occupations across all field sites are: Administrator, Deputy Practice Manager, General Practitioner, Healthcare Assistant, Nurse Practitioner, Pharmacy Technician, 
Phlebotomist, Practice Manager, Practice Nurse, Practice Pharmacist, Prescription Clerk, Receptionist, Secretary, and Summarizer. Because this article concerns the documentation work of GPs specifically, the field researcher observed and attended clinics of 12 GPs. However, other occupations such as practice nurses, physician's assistants, nurse practitioners, and to some extent healthcare assistants also document clinical encounters with the patient.

Interviews were semi-structured, and questioning took place while the participant was observed working on their daily routine or showing the field researcher different tasks. At some points the field researcher would hold on asking questions or interacting with participants if critical events occurred that required immediate focus and attention of the participant. At the end of fieldwork at each site the field researcher would conduct a focus group open to all practice staff. The conversation of the focus group was on verifying and member checking the accuracy of data gathered about tasks observed, and to have a discussion on the use of automation and artificial intelligence at that practice.

The field researcher was present during clinical appointments to observe the clinician when patients consented to the presence of the researcher. During clinical appointments the field researcher sat in the corner of the room watching the general practitioner or other clinicians, taking notes on how they work and what tools they use during patient appointments. Follow up questions during the clinical encounter were written down and asked to the provider after the patient exits the examination room. Additionally, at each field site GPs have a coffee break, or case study, where all GPs available would gather in the break room or similar meeting room to discuss difficult patient cases, advice on documentation, or mentor other GPs in training. The field researcher was invited to, and attended, these coffee break case study meetings where often additional documentation, writing, and conversation occurred.

While in the field documents that describe occupational responsibilities or work tasks were gathered, along with photos or sample of any other documents, tools, or software that clarify or represent the work a staff member engages. The field researcher wrote detailed field notes of observations and during questions and interviews. Then, the researcher developed a spreadsheet of each occupation and a list of the tasks they perform. These data were used at subsequent field observations and shown to the same occupational type at different primary care centers to help confirm each tasks representativeness and accuracy.

In addition to member checking and focus groups for establishing trustworthiness in this study, findings and fieldwork experiences were regularly reported and discussed with the project advisory board. The project advisory board membership includes two general practitioners and two policy stakeholders with experience in NHS and in primary care. The field researcher also produced an audit trail through memo writing and keeping a detailed research log data file.

Task data were coded in the spreadsheet and further enriched through a series of categories that include the frequency of task, any tools used, type of software required, and other features of the task including conceptual inductive categories. Analysis for this paper focused on the documentation practices of GPs. Important themes discussed 
in the findings sections were elicited and refined after a continuous process of conversation and collaborative sensemaking between the researchers.

\section{$3 \quad$ Findings}

A task or series of tasks that form a critical part the general practitioners (GP) work pertaining to documentation of the clinical encounter. Writing notes in the patient's electronic medical record ranges from simple and quick to complex and time consuming. The complexity and length of time it takes a GP to document the patient encounter depends on what the patient is being seen for, the prior relationship between the patient and provider, when they were last seen, and even the experience of the provider. This can amount to writing a few codes - a standardized medical taxonomy for diagnosis and a sentence or two that captures the patient's current appointment, progress, change in therapy, or anything else clinically relevant about the appointment. Or, the documentation work can involve multiple codes, referencing information in the British National Formulary, looking up information online, studying lab work, revising previously written text, calling a specialist for advice, and writing a detailed multi paragraph account of the consultation.

Our fieldwork suggests this important work must be done promptly. The time GPs have to document is from when the patient exits the examination room, to when the next patient is called to see the provider. Several minutes at most. It is considered by many GPs involved in this study a best practice to finish documentation work right after the patient's appointment, not letting it wait till the end of the day.

The interpersonal style with which GPs write ranges from not looking directly at the patient while loudly banging away on the keyboard typing rapidly as patients discuss their concerns; to the GP directly facing the patient, hands on lap, listening and discussing them and only writing on the computer when their leave or near the end of the appointment. We observed GPs incorporate the computer into the clinical appointment in different ways, some minimize its presence and others bring the computer in as a third member to reference information and write notes. This use, too, is influenced by the context for the patient's appointment, how friendly the patient and provider are with each other, and the clinicians experience.

Every primary care practice in this study but one uses an electronic medical record called EMIS. Although its functionality is too large to detail here, the section of the record most pertinent are the patient notes. The record has preformatted sections for notes that are typically organized in the following structure: problem, history, examination, family history, social, comment, medication, follow up, procedure, test request, referral, document, and allergy. Not all these sections are used, and GPs exercise their own authorial voice and style when documenting their patients. In fact, several experienced GPs were able to discern what notes from a new patient were written by locums or other staff such as a nurse or physicists assistant.

We observed that during a chronic disease clinic one GP kept two patient notes open, thinking about one complex situation while they worked on the most recent patient's documentation notes. This allowed them to mull over the patient's case and ask some 
advice from their colleague as they considered the pros and cons of treatment options. The GP had captured the core of what the appointment was about. The remaining documentation work was the careful and considerate work that comes with the practicing of medicine. The decisions to continue or change this patients therapy was a decision that needed to be personalized to that patient's context, not only the patient's specific health situation but also their families concern, the patient's own considerations, and the informed decision that comes with medical treatment. It was through this event that the personal preferences in documentation work become apparent. The need to carefully consider a case is shown through its persistence on the clinician's screen, not to have notes quickly typed and closed.

Another important observation the field researcher made is the use of templates that come with the medical record. The GP attending to their clinical hours receives suggestions for what templates to use when seeing a patient. The suggested template is based on what the patient is being seen for and is a nationally standardized template. These templates automatically pop up through a notification and offer a suggestion that will pre-populate some of the documentation fields, similar to auto-complete word suggestions when texting on a mobile phone. These popups and suggestions were ignored every time by this clinician. In favor of their own personal style to document by creating a notes section in the patients record and writing the following sections: problem, history, and open comment. The GP will use discretion to write a few notes in the record during the clinical appointment but complete the rest of their documentation work when the patient leaves.

The work of documenting clinical encounters is complicated and highly situated, constantly negotiated by the patient and provider we studied. As noted, researchers have presented this experience to be automated. There are smart technologies that currently capture a natural language discussion between two people. Most all consumer smart phones, the current flood of smart speakers and other smart devices have speech recognition functionality. High quality speech recognition and transcription has been available on consumer smart phones for years.

It is reasonable to consider the use of a speech recognition device that can turn the patient and provider discussion into a text transcript. Using natural language processing this text could be further reduced and annotated to provide clinically relevant information that matches the way the provider writes or is categorized by the previously mentioned sections of the medical record.

Connected to the electronic medical record, this device could utilize data already in the patients' record as well as similar patients at this practice, providing the technology with data to learn from and support the providers' decision making and documentation responsibilities. After the clinical encounter the clinician reviews a summary they can edit and save to the patients file. Almost the entire process would be automated, invisible, and would allow the provider to interact directly with the patient, not having to consider documenting the encounter.

This scenario was presented at each focus group and provided lengthy discussion during several interviews with clinicians. This potential solution to automate the documentation of the clinical encounter was seen by many providers as useful, including 
many "when can we have that" comments. While technically possible and able to reduce cumbersome documentation work, this is a set of tasks that if made invisible through automation would have negative consequences on the clinical encounter and even undermine the skills and problem-solving ability of the clinician.

One GP critiqued an AI generating an entire transcript of the patient and providers conversation with "I don't need the entire transcript, it's not useful or clinically relevant to me." It was seen as a flood of information. Not everything being relevant to the diagnosis, treatment, or continuing therapy for the patient.

It was observed that it was not uncommon for GPs to rewrite, backspace, or edit the notes they wrote, particularly notes for new cases or complex circumstances, particularly mental health encounters, as well as newer or training GPs. What looks like GPs writing down notes on a computer is actually a medically trained clinician dealing with qualitative data (clinical narrative) through the process of writing to themselves and to other medical professionals: an act they spent years at school and training for to think in a critical way about medicine and treatment.

If the practice of writing and thinking through writing is wholly removed from the clinicians' workflow then it removes an opportunity for the clinician to apply the skills that they have trained over years of a medical education, and it removes an opportunity for the clinician to think and reflect critically, in the way they practice medicine. Research shows that writing engages the brain, allows GPs to be better observers, supports empathy, and engages critical thinking [15].

\section{Discussion}

Findings presented here offer a deeper and situated perspective into the documentations activities of GPs. These activities can be understood as information practices, which are defined as socially and culturally established ways of identifying, seeing, using and sharing the information [16]. Information practices reflect not only individual choices that each GP makes but also his or her encounter with the social context within which the documentation is done, particularly the social interactions with the patient and other medical professionals. Bansler et al [2] similarly highlight differences between personal and social context of clinical documentation practices; they suggest that documentation can serve as "tool of thinking" for doctors and as an artifact of coordination and communication with other involved parties.

In addition, documentation as an information practice involves both manipulation of explicit information artifacts (e.g., the patient digital record) and tacit and embodied interactions with the information environment (e.g., subjective ways to make sense of patients' data and communicate it to the patient) [17]. Intelligent technological systems are likely to capture and automate the process of manipulating and analyzing the information artifacts; new AI systems will provide decision support to medical professionals based on their superior capabilities in searching and analyzing big data and comparing patients' data with previous cases. However, the tacit and more contextual dimension of the information practice of documentation such as reading social cues and combining them with decisions suggested by AI to communicate with patients continues to lie 
outside of the capabilities of intelligent systems and are best handled by humans. Automating the whole documentation process by implementing AI systems as advocated by many technologists and some academic researchers is shortsighted and tends to gloss over the intricacies of situated practices that we outlined in this paper. In fact health informatics researchers and electronic health record vendors for years have sought to invoke smart technologies to transform doctor's note into structured content; however, these notes include clinical narratives, which serve different purposes such as collective sensemaking by medical professionals [2].

Below (Fig. 1), we present a bisection of our empirical investigation of clinical documentation work, with suggestions on the most advantageous configurations that leverages the strengths of healthcare providers and artificial technologies. This builds on the concept of human-AI symbiosis; suggesting human and AI serve complementary roles based on their unique and irreplaceable capabilities [18].

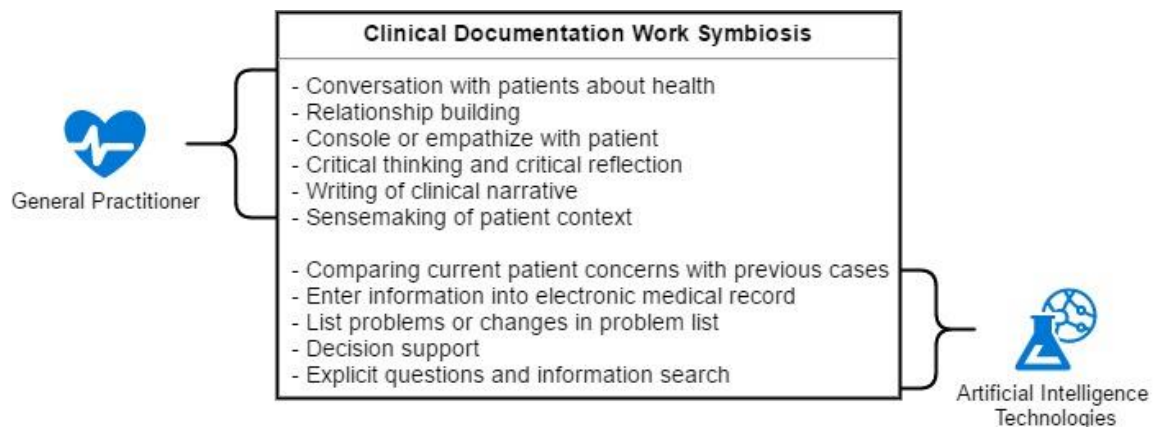

Fig. 1. List of documentation tasks best performed by clinicians and AI technologies.

New machine and deep learning algorithms provide avenues for automating tasks that were done exclusively by humans. However, the vision of human-AI symbiosis involves a normative stance: the fact that many tasks can be automated does not necessarily mean they should [9]. This runs against the old trope of automation, which implicitly espouses automating whatever that can be automated. Such technological imperatives grossly overlook the complexity of healthcare sociotechnical systems including people roles, sensemaking practices and the nature of human interaction and care. Human-AI symbiosis implies that more effective (not necessarily more efficient) practices in healthcare will likely exhibit a synergetic partnership between medical professionals and AI systems.

The symbiosis also means as more advanced AI algorithms are developed and introduced into healthcare, health professionals must adapt and develop new skills and competencies. To keep a balanced division of work between AI and humans, doctors and other medical professionals must continuously upgrade skills that are considered competitive edge of humans (such as those listed in Fig. 1). In addition, medical professionals need to acquire literacies on how to harness the capabilities of intelligence systems in their work practices, what Jarrahi [18] calls "AI literacies". 


\section{Conclusions}

In this paper we explore the practice of documentation work in primary care as performed by general practitioners. We posit that, while important work that should be automated to avoid GP burnout and stress, this is a task that should not be wholesale automated. Our question on what problems do general practitioners clinical documentation work practices create for automation by AI? Has yielded several challenges around individual clinician style, tradition, patient context, tacit information, and the practice of writing enabling critical thought and critical reflection. We push back on the idea that this set of tasks and work practices can be automated wholesale. While AI technologies are capable to automate most of the tasks involved in the workflow of clinical documentation, we raise the concern that this new configuration of deep learning and AI technologies, while impressive in their ability to mimic and augment thinking, decision making, and knowledge work, will change the clinician's ability to think critically, reflect, and practice medicine effectively. We side on the concept of augmenting GP documentation work and provide specific areas of documentation work that best suit the strengths of GPs and AI technologies, while preserving the critical thinking, and reflection, tacit work, and GPs interactions with their patients.

\section{References}

1. Sola, D., Borioli, G.S., Quaglia, R.: Predicting GPs' engagement with artificial intelligence. Br. J. Healthc. Manag. 24, 134-140 (2018).

2. Bansler, J.P., Havn, E.C., Schmidt, K., Mønsted, T., Petersen, H.H., Svendsen, J.H.: Cooperative Epistemic Work in Medical Practice: An Analysis of Physicians' Clinical Notes. Comput. Support. Coop. Work. 25, 503-546 (2016).

3. Lin, S.Y., Shanafelt, T.D., Asch, S.M.: Reimagining Clinical Documentation With Artificial Intelligence. Mayo Clin. Proc. 93, 563-565 (2018).

4. Verghese, A., Shah, N.H., Harrington, R.A.: What This Computer Needs Is a Physician: Humanism and Artificial Intelligence. JAMA. 319, 19 (2018).

5. Baird, B., Charles, A., Honeyman, M., Maguire, D., Das, P.: Understanding pressures in general practice. , London, UK (2016).

6. Hopson, C.: The sate of the NHS provider sector. (2016).

7. Byrne, L., Bottomley, J., Turk, A.: British Medical Association Survey of GPs in England. , London (2016).

8. Gibson, J., Checkland, K., Coleman, A., Hann, M., McCall, R., Spooner, S., Sutton, M.: Eighth National GP Worklife Survey. , Manchester (2016).

9. Zuboff, S.: In the Age of the Smart Machine: The Future of Work and Power. Basic Books, New York (1988).

10. Smith, K., Smith, V., Krugman, M., Oman, K.: Evaluating the Impact of Computerized Clinical Documentation. Comput. Informatics, Nurs. 23, 132-138 (2005).

11. Ammenwerth, E., Spötl, H.-P.: The time needed for clinical documentation versus direct patient care. A work-sampling analysis of physicians' activities. Methods Inf. Med. 48, 84-91 (2009). 
12. Erickson, S.M., Rockwern, B., Koltov, M., McLean, R.M., Medical Practice and Quality Committee of the American College of Physicians: Putting Patients First by Reducing Administrative Tasks in Health Care: A Position Paper of the American College of Physicians. Ann. Intern. Med. 166, 659 (2017).

13. Rule, A., Rick, S., Chiu, M., Rios, P., Ashfaq, S., Calvitti, A., Chan, W., Weibel, N., Agha, Z.: Validating free-text order entry for a note-centric EHR. AMIA ... Annu. Symp. proceedings. AMIA Symp. 2015, 1103-10 (2015).

14. Klann, J.G., Szolovits, P.: An intelligent listening framework for capturing encounter notes from a doctor-patient dialog. BMC Med. Inform. Decis. Mak. 9 Suppl 1, S3 (2009).

15. Wald, H.S., Borkan, J.M., Taylor, J.S., Anthony, D., Reis, S.P.: Fostering and Evaluating Reflective Capacity in Medical Education: Developing the REFLECT Rubric for Assessing Reflective Writing. Acad. Med. 87, 41-50 (2012).

16. Savolainen, R.: Everyday information practices: A social phenomenological perspective. Scarecrow Press (2008).

17. Jarrahi, M.H., Thomson, L.: The interplay between information practices and information context: The case of mobile knowledge workers. J. Assoc. Inf. Sci. Technol. 68, 1073-1089 (2017)

18. Jarrahi, M.: Artificial Intelligence and the Future of Work: Human-AI Symbiosis in Organizational Decision Making. Bus. Horiz. (2018). 